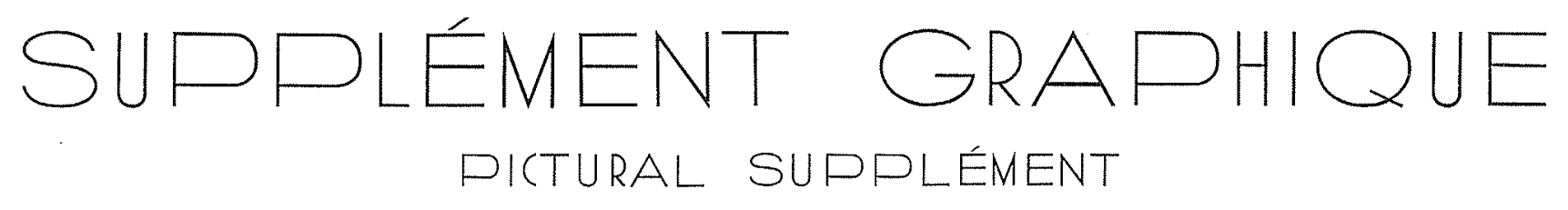

L y a un on, nous annoncions à nos lecteurs notre intention de consacrer un numéro hors série à l'usine-barrage de Génissiat sur le Rhône, dont la mise en service progressive était déjà largement commencée.

Cet ouvrage est maintenont paru et c'est de ses pages que nous avons extrait les quelques clichés qui constituent ce "Supplément graphique ».

Dans la vie « hydraulique " française, Génissiat a tenu une place suffisamment importante pour que, dans l'un de ses numéros normaux, "la Houllle Blanche" lui consacre quelques pages, ne seraitce que sous forme d'images.

Nos lecteurs retrouveront ci-crprès les principaux aspects de cette œuvre grandiose; du même coup, ils pourront se faire une idée de la présentation donnée à notre numéro spécial, où 225 figures du genre de celles qui suivent illustrent un texte dont nous croyons devoir reproduire ci-dessous le sommaire:

-.- Statut, passé et présent de la Compagnie Nationale dul Rhöne (G. TOUnNinr).

\section{LA CONCEPTION DE GENISSTAT}

- L'élaboration du projet (MI. HevnY).

- Les conditions géologiques de l'aménagement hydro-électrique. du Rhône entre Genève et Seyssel (MI. GrgNOUX et J. MATHAaN).

\section{LE GENIE CIVIL}

- Le Génic civil de Génissiat. Vac d'ensemble (G. Grès).

- Les tranuan préliminaires du barrage de Génissial (Ch. Cragnaud).

- Les lravaux définitifs (H. Escalon et H. Diserexs).

\section{L'EQUIPEMENT'}

-..- L'équipement hydro-électrique el mécanique de la centale Léon Perrié, à Génissiat (A. RoвERT).

- Les conduites forcées de Génissiat

(G. FERRAND).

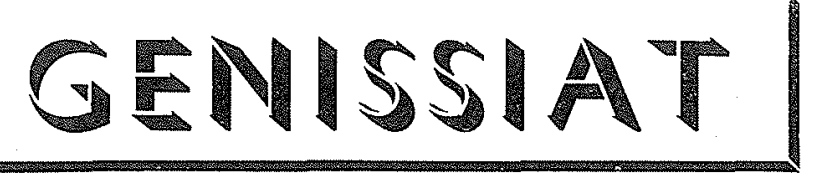

- Vannes-papillon des turbines (โ. ARManef). - Quelques problèmes posés par l'étude et la construction des turbines de Génissiat (J. AnNouD et A. Suss).

- Les vannes de Génissiat (H. Gérodolde et F. Mussard).

- L'exploitation de Génissiat et l'avenir de l'aménagement du Rhóne (P. DeuatTre).

- Bibliographie sommaire de Génissiat.

Pour être complet, nous jugeons utile de rappeler ci-dessous les principales caractéristiques de Génissiat :

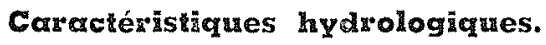

Bassin versant. . . . . . . . . . . .

Débit minimum instantané. . . . . . .

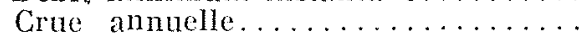

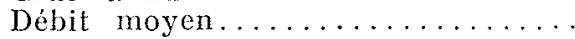

Plus forte crue connue..........

$10.910 \mathrm{~km}^{2}$ $100 \mathrm{~m} 2 / \mathrm{s}$ $737 \mathrm{~m}^{2} / \mathrm{s}$ $379 \mathrm{~m}^{3} / \mathrm{s}$ $2.000 \mathrm{~m} \mathrm{~m}^{3} / \mathrm{s}$

\section{Barrage.}

- Type poids, incurvé en plan (rayon : $300 \mathrm{~m}$ ), de $104 \mathrm{~m}$ de hautenr et de $140 \mathrm{~m}$ de longueur au couronnement (béton mis en ounve: plus de $\left.400.000 \mathrm{~m}^{3}\right)$.

- 6 conduites forcées de $5,75 \mathrm{~m}$ de diamètre, noyées dans la masse du barrage.

\section{Evacuation des crues.}

1 évacuateur superficiel R.D. débit maximum............ $2.700 \mathrm{~m}^{3} / \mathrm{s}$

1 évacuateur souterrain R.G. : débit maximum.......... $1.300 \mathrm{~mm} / \mathrm{s}$

$$
\text { AU TOTAL...... } \overline{4.000 \mathrm{~m}^{3} / \mathrm{s}}
$$

Usine. Prévue pour 6 groupes.

Actuellement :

4 groupes en service.

1 groupe en cours d'installation.

1 groupe en attente.

Turbines : Type Francis a axe vertical :

$90.000 \mathrm{ch}$ sous $64,50 \mathrm{~m}$ de chute (débit correspondant: environ $120 \mathrm{~m} / \mathrm{s}$ ); vitesse normale : 150 trs $/ \mathrm{mn}$.

Alternateurs :

$$
65.000 \mathrm{~kW}-70.000 \mathrm{kVA}-15 \mathrm{kV} \text {. }
$$

Transformateurs :

1 transformateur par groupe $15-220 \mathrm{kV}$ on $15-150 \mathrm{kV}$ (70.000 kVA)

\section{Production annuelle.}

1,7.109 $\mathrm{k}$ Wh en année moyenne, avec cinq groupes. (Fhoto V. Cuyl, Lyon) 


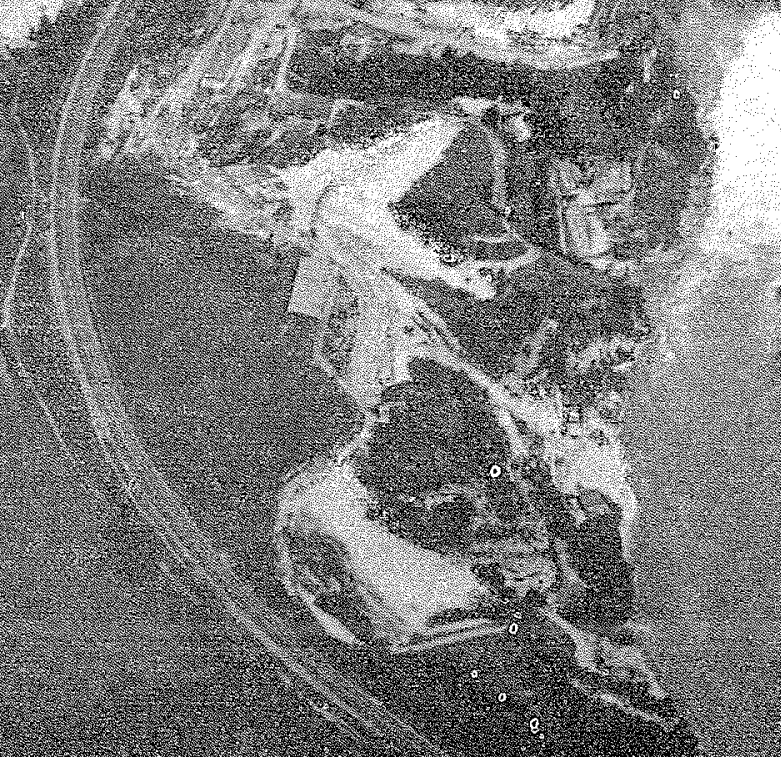




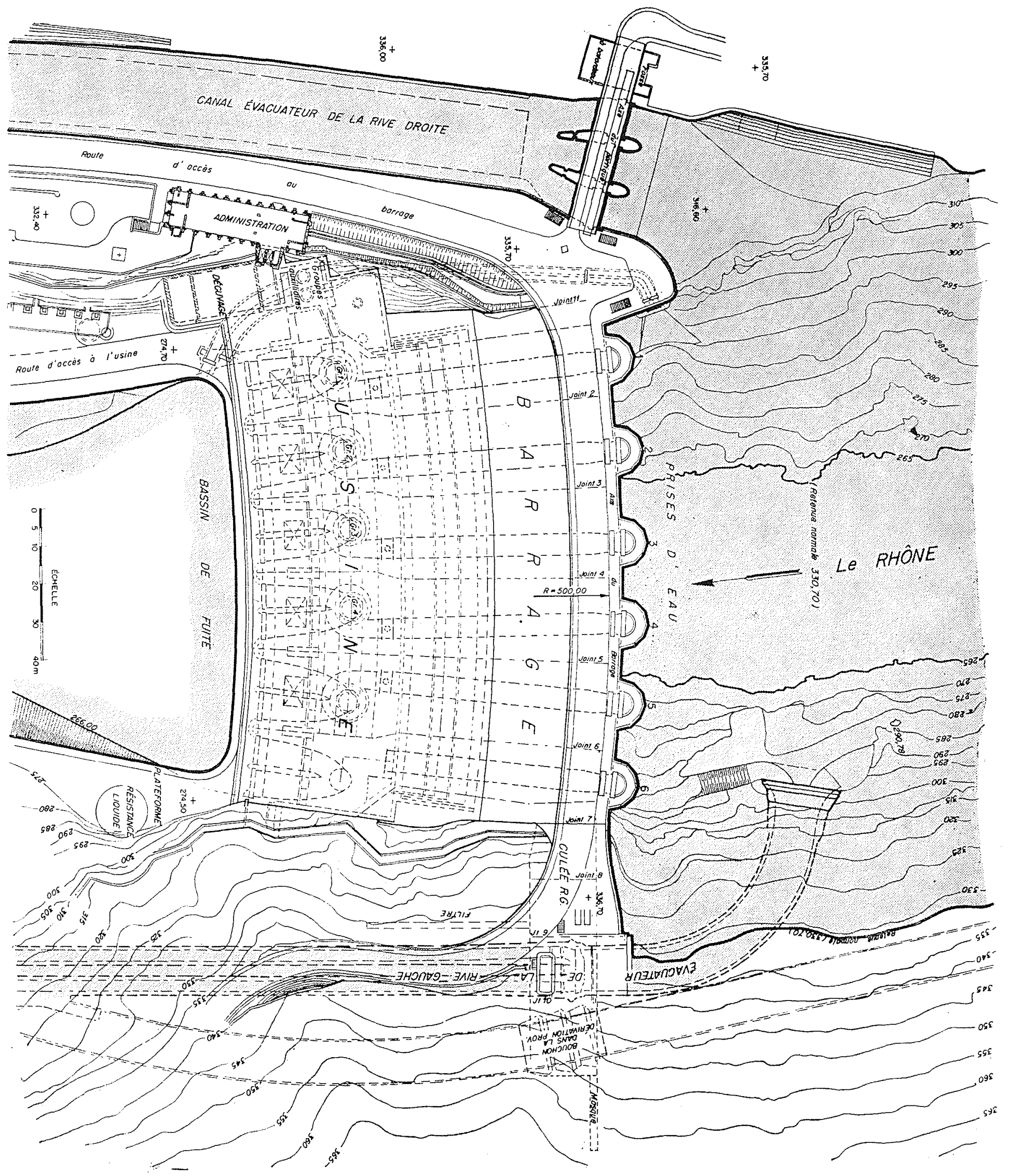




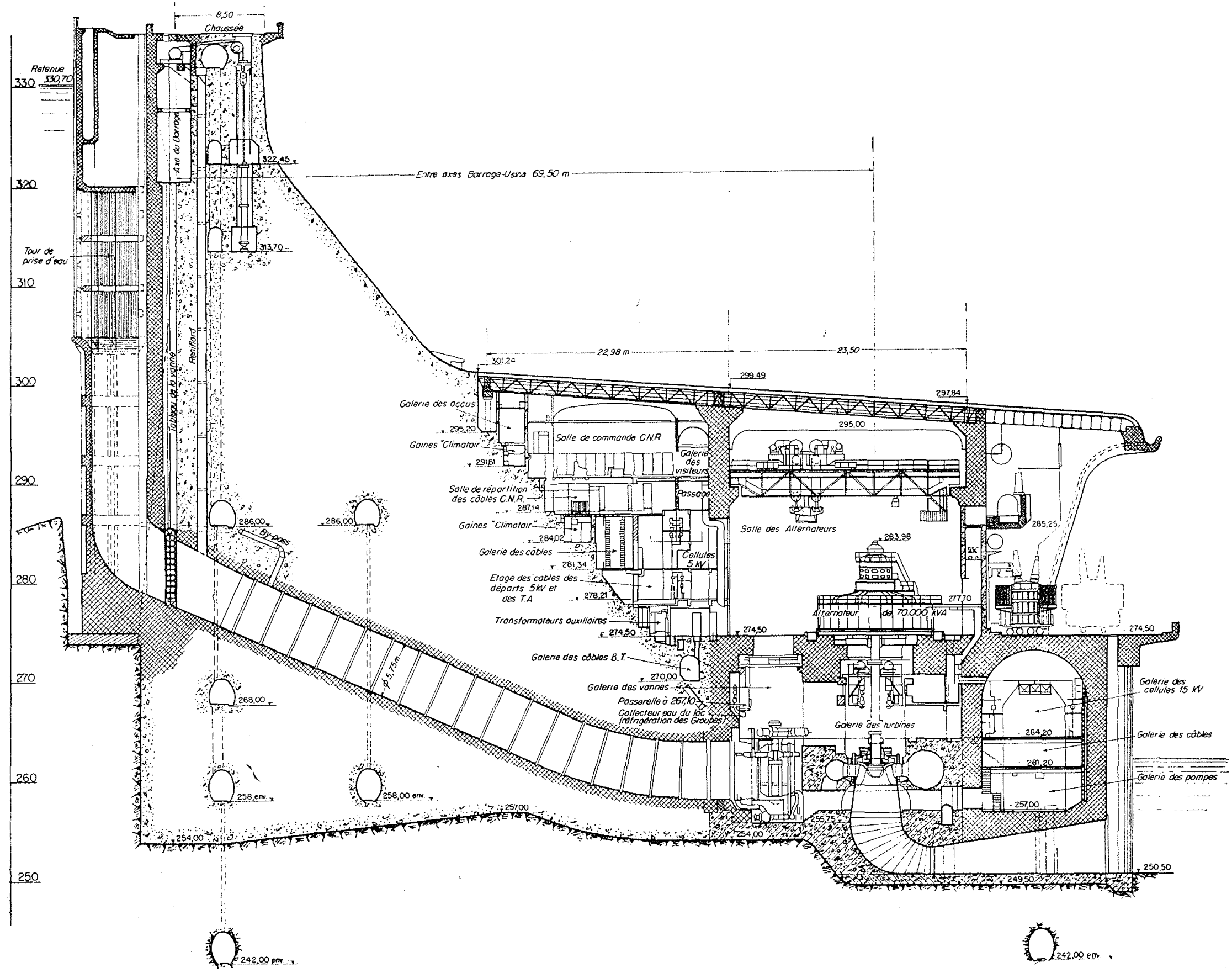

En haut: Plan d'ensemble du barrage et de l'usine.

En bas: coupe transversale du barrage et de l'usine. 


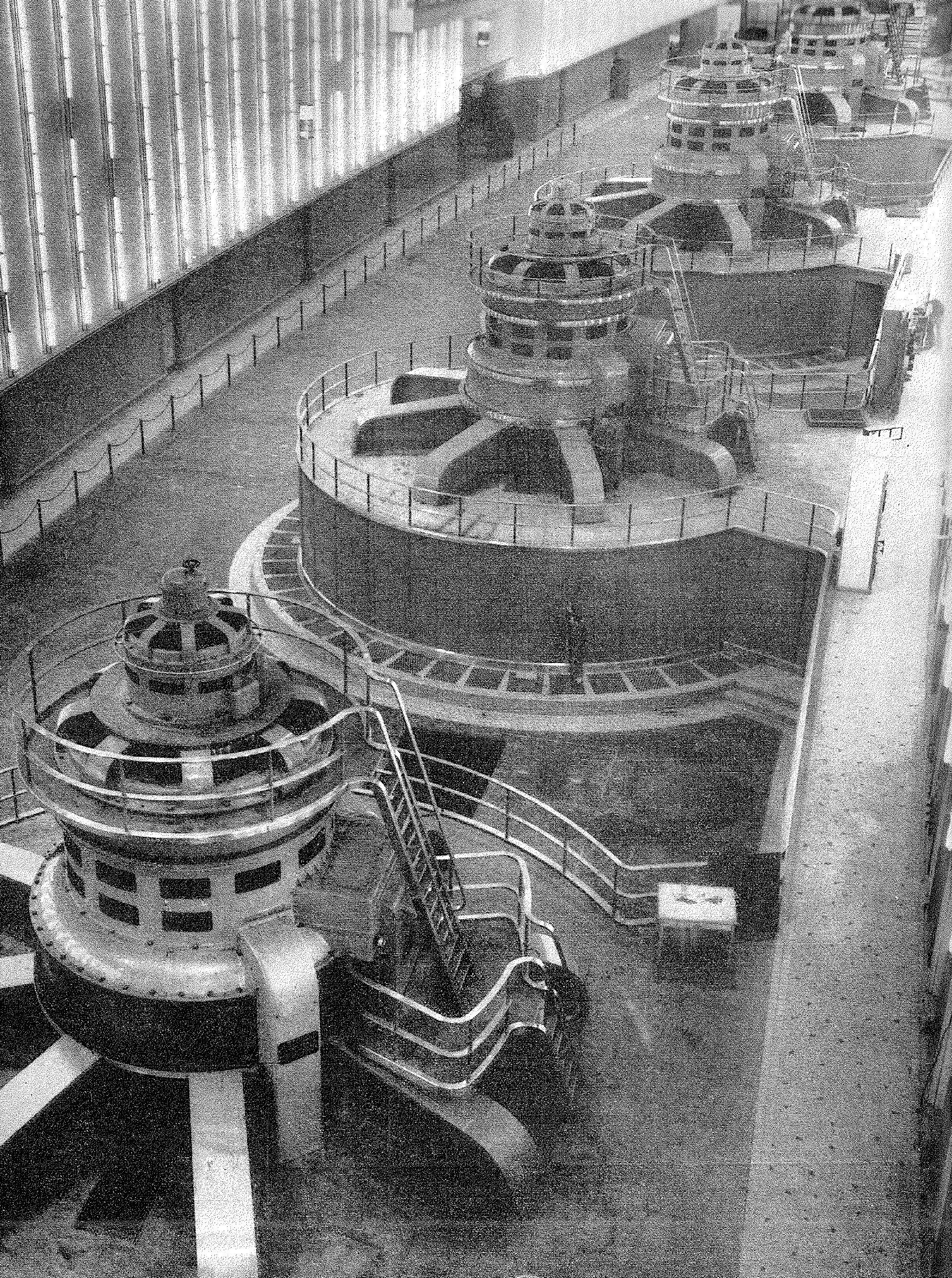




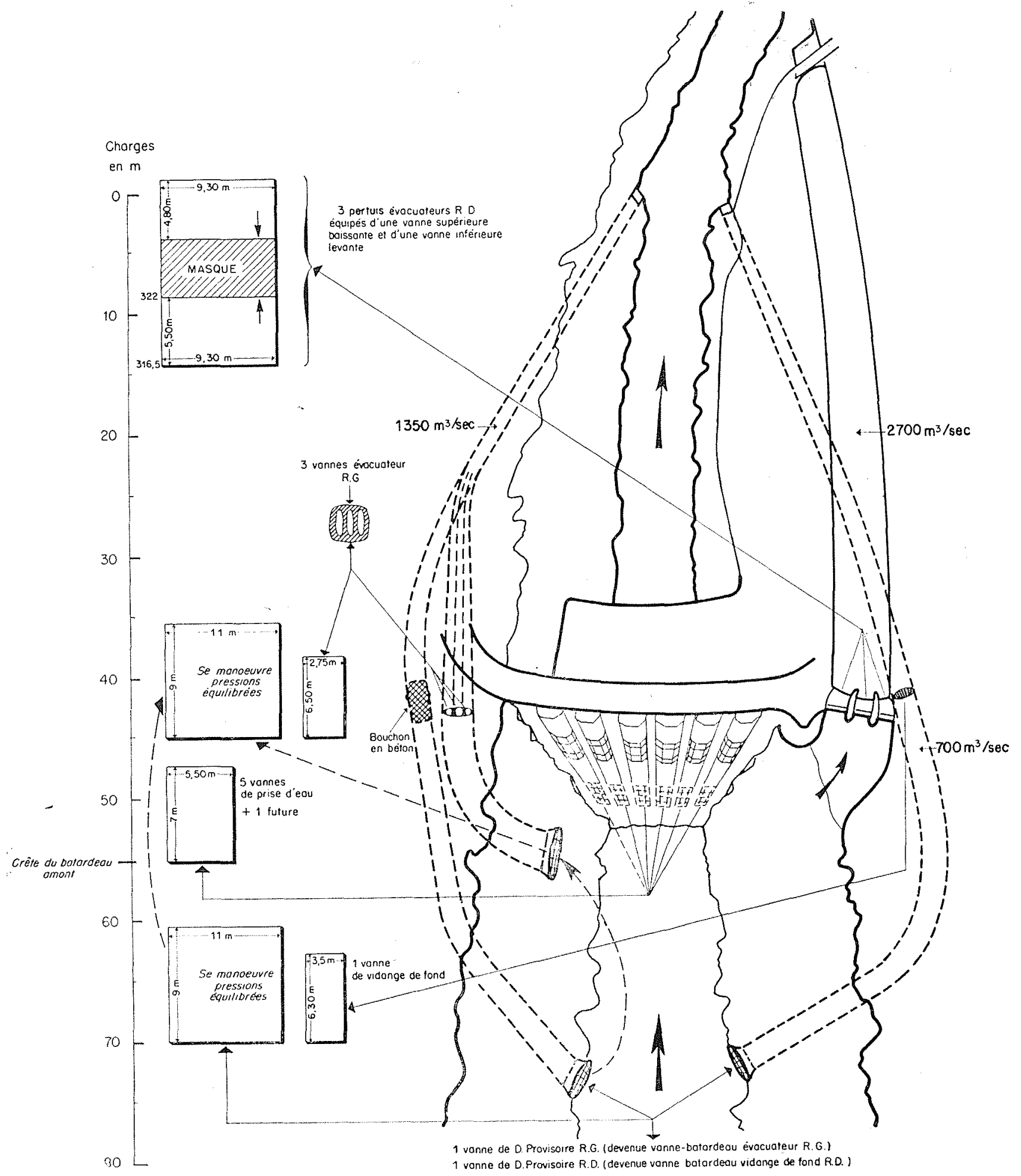

Les vannes de Génissiat. - Schéma d'implantation. 


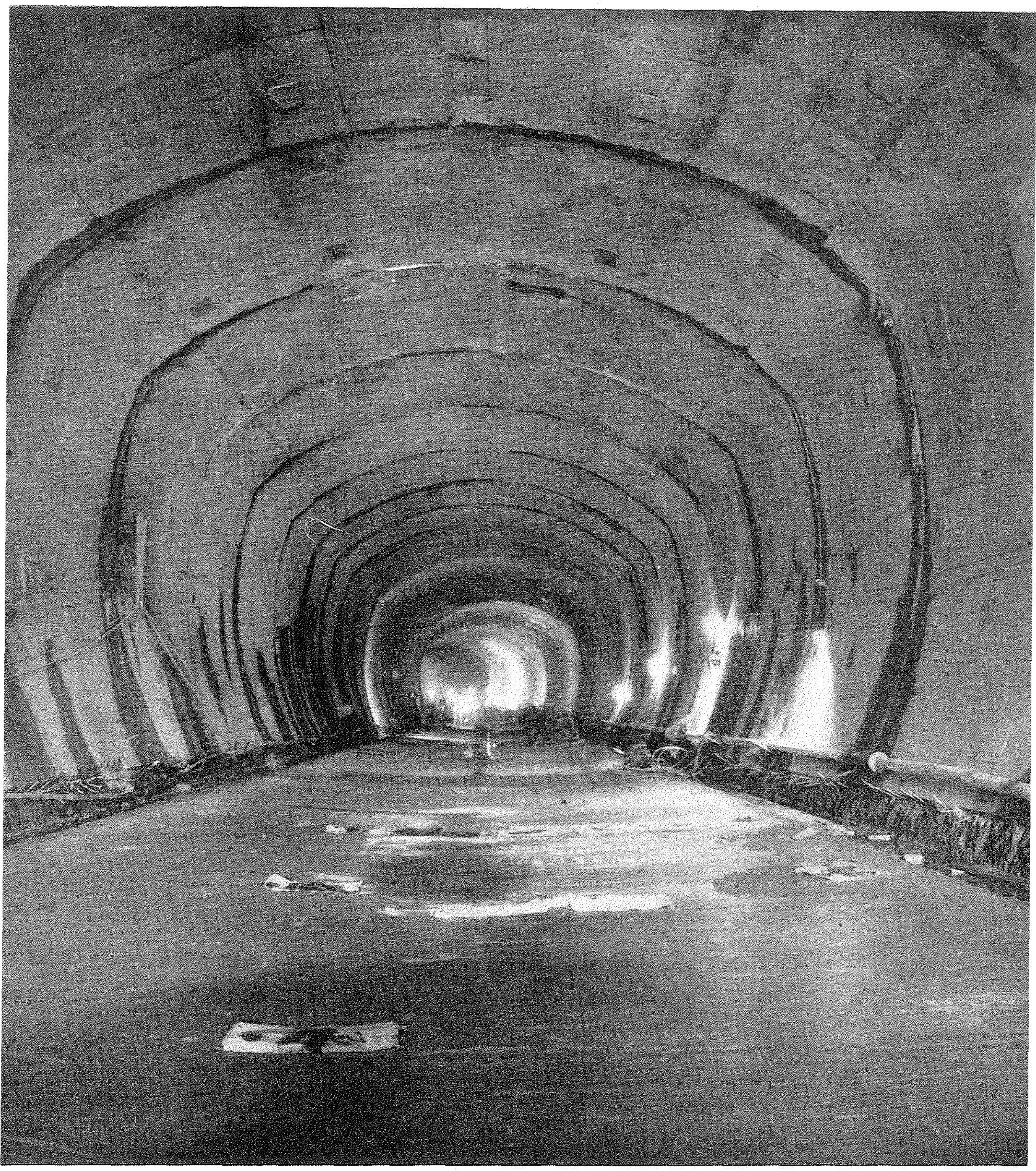




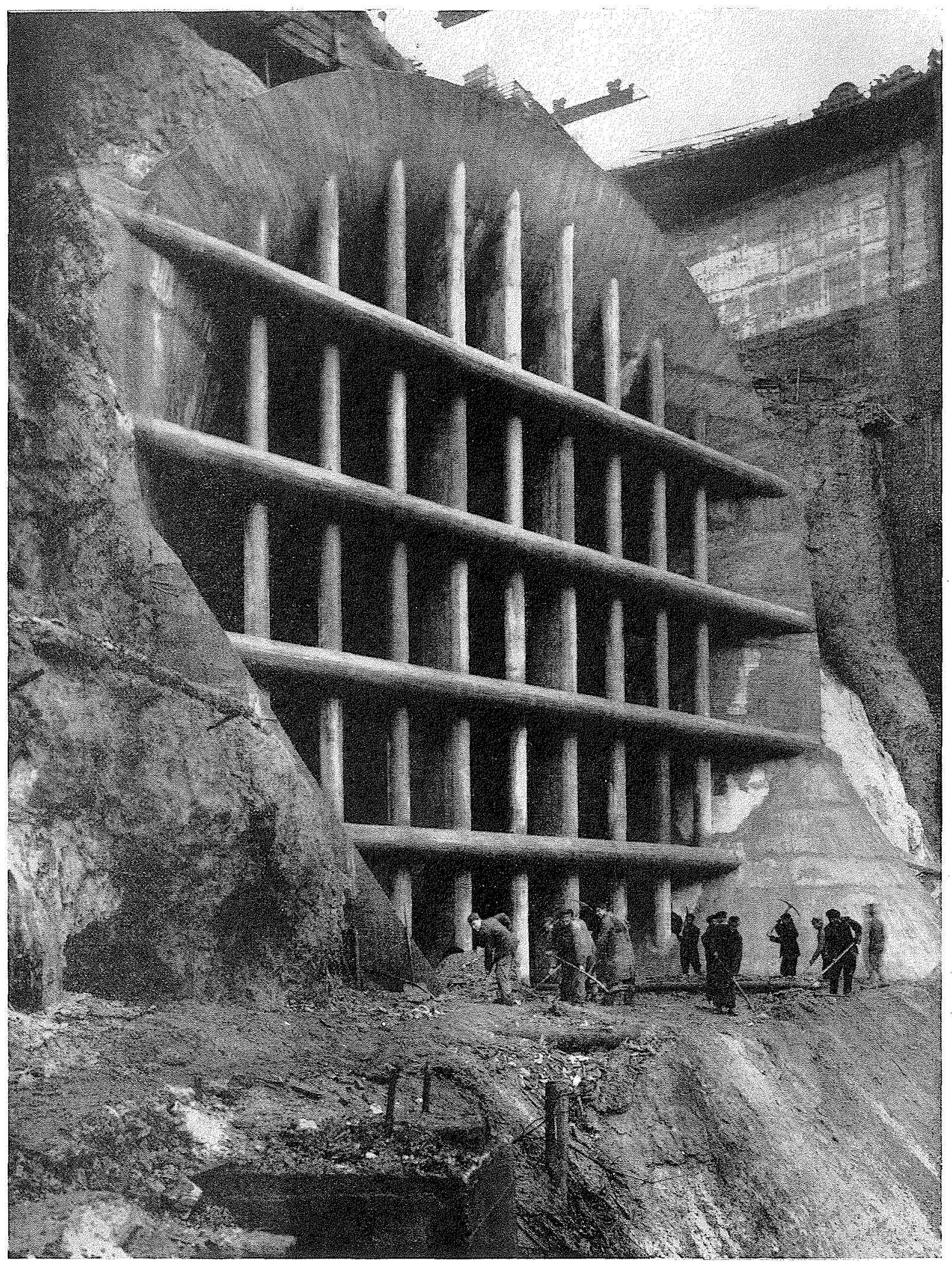

(Cliché Boyer) 


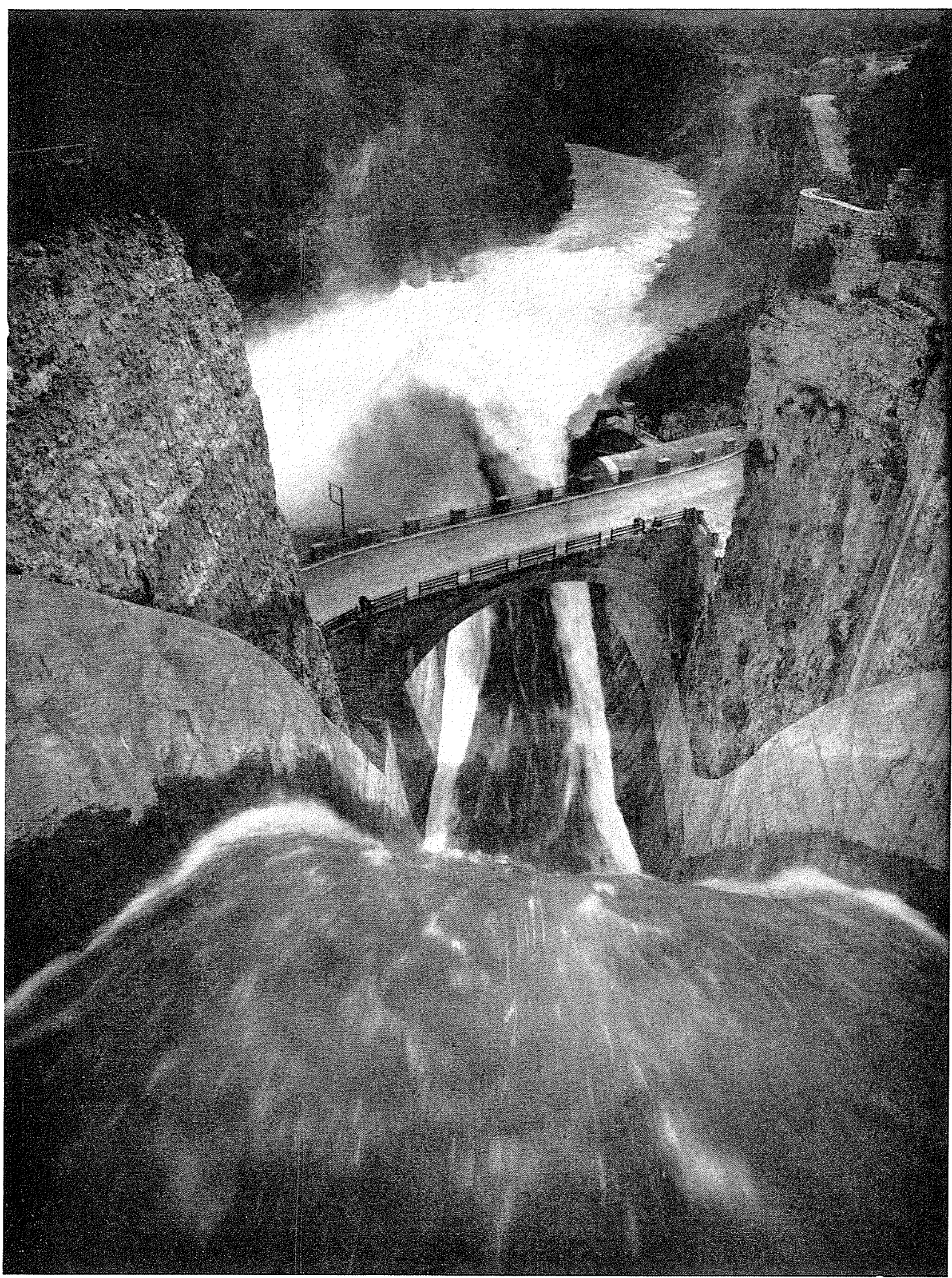

